\title{
Article
}

\section{Improved Protoporphyrin IX-Guided Neurosurgical Tumor Detection with Frequency-Domain Fluorescence Lifetime Imaging}

\author{
David Reichert ${ }^{1,2} \oplus$, Mikael T. Erkkilae ${ }^{1} \oplus$, Johanna Gesperger ${ }^{1,3}\left(\mathbb{C}\right.$, Lisa I. Wadiura ${ }^{4} \oplus$, Alexandra Lang ${ }^{4} \oplus$, \\ Thomas Roetzer-Pejrimovsky $^{3}{ }^{\circledR}$, Adelheid Woehrer ${ }^{3}{ }^{(}$, Marco Wilzbach ${ }^{5}$, Christoph Hauger ${ }^{5}$, Wolfgang Drexler ${ }^{1}(\mathbb{D}$,

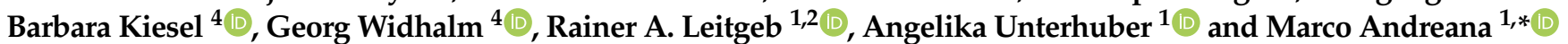

1 Center for Medical Physics and Biomedical Engineering, Medical University of Vienna, 1090 Vienna, Austria; david.reichert@meduniwien.ac.at (D.R.); mikael.erkkilae@web.de (M.T.E.); johanna.gesperger@meduniwien.ac.at (J.G.); wolfgang.drexler@meduniwien.ac.at (W.D.); rainer.leitgeb@meduniwien.ac.at (R.A.L.); angelika.unterhuber@meduniwien.ac.at (A.U.)

2 Christian Doppler Laboratory OPTRAMED, Medical University of Vienna, 1090 Vienna, Austria

3 Division of Neuropathology and Neurochemistry, Department of Neurology, Medical University of Vienna, 1090 Vienna, Austria; thomas.roetzer@meduniwien.ac.at (T.R.-P.); adelheid.woehrer@meduniwien.ac.at (A.W.)

4 Department of Neurosurgery, General Hospital and Medical University of Vienna, 1090 Vienna, Austria; lisa.wadiura@meduniwien.ac.at (L.I.W.); alexandra.lang@meduniwien.ac.at (A.L.); barbara.kiesel@meduniwien.ac.at (B.K.); georg.widhalm@meduniwien.ac.at (G.W.)

5 Advanced Development Microsurgery, Carl Zeiss Meditec AG, 73447 Oberkochen, Germany; marco.wilzbach@zeiss.com (M.W.); christoph.hauger@zeiss.com (C.H.)

* Correspondence: marco.andreana@meduniwien.ac.at; Tel.: +43-1-40400-39223

\section{check for} updates

Citation: Reichert, D.; Erkkilae, M.T.; Gesperger, J.; Wadiura, L.I.; Lang, A.; Roetzer-Pejrimovsky, T.; Woehrer, A.; Wilzbach, M.; Hauger, C.; Drexler, W.; et al. Improved Protoporphyrin IX-Guided Neurosurgical Tumor Detection with Frequency-Domain Fluorescence Lifetime Imaging. Appl. Sci. 2022, 12, 1002. https://doi.org/ 10.3390/app12031002

Academic Editor: Kijoon Lee

Received: 29 November 2021

Accepted: 14 January 2022

Published: 19 January 2022

Publisher's Note: MDPI stays neutral with regard to jurisdictional claims in published maps and institutional affiliations.

Copyright: (C) 2022 by the authors. Licensee MDPI, Basel, Switzerland. This article is an open access article distributed under the terms and conditions of the Creative Commons Attribution (CC BY) license (https:// creativecommons.org/licenses/by/ $4.0 /)$.

\begin{abstract}
Precise intraoperative brain tumor visualization supports surgeons in achieving maximal safe resection. In this sense, improved prognosis in patients with high-grade gliomas undergoing protoporphyrin IX fluorescence-guided surgery has been demonstrated. Phase fluorescence lifetime imaging in the frequency-domain has shown promise to distinguish weak protoporphyrin IX fluorescence from competing endogenous tissue fluorophores, thus allowing for brain tumor detection with high sensitivity. In this work, we show that this technique can be further improved by minimizing the crosstalk of autofluorescence signal contributions when only detecting the fluorescence emission above $615 \mathrm{~nm}$. Combining fluorescence lifetime and spectroscopic measurements on a set of 130 ex vivo brain tumor specimens (14 low- and 56 high-grade gliomas, 39 meningiomas and 21 metastases) coherently substantiated the resulting increase of the fluorescence lifetime with respect to the detection band employed in previous work. This is of major interest for obtaining a clear-cut distinction from the autofluorescence background of the physiological brain. In particular, the median fluorescence lifetime of low- and high-grade glioma specimens lacking visual fluorescence during surgical resection was increased from $4.7 \mathrm{~ns}$ to $5.4 \mathrm{~ns}$ and $2.9 \mathrm{~ns}$ to $3.3 \mathrm{~ns}$, respectively. While more data are needed to create statistical evidence, the coherence of what was observed throughout all tumor groups emphasized that this optimization should be taken into account for future studies.
\end{abstract}

Keywords: fluorescence-guided surgery; fluorescence lifetime imaging; fluorescence spectroscopy; protoporphyrin IX

\section{Introduction}

In the United States, the average annual age-adjusted incidence rate of patients diagnosed with a tumor of the central nervous system (CNS) is 24 per 100,000 [1]. Particularly, infiltrative gliomas have a poor prognosis. Median survival ranges from about 8 to 15 months for glioblastoma multiforme (GBM) compared to 3 to 10 years for low-grade gliomas (LGG) [1,2]. Maximizing the extent of resection while prioritizing neurological function in cytoreductive surgery is one of the most decisive prognostic factors and essential in the initial treatment of gliomas, meningiomas (MNG) and brain metastases (MET) [3-9]. Protoporphyrin IX (PpIX) fluorescence guidance was first established for 
intraoperative visualization of high-grade gliomas (HGG) $[10,11]$ and was afterwards investigated in MNG [12] and MET [13], with promising results. Presurgical administration of the non-fluorescent precursor 5-aminolevulinic acid (5-ALA) leads to tumor-specific metabolization of PpIX [14]. Surgical microscopes can be equipped with optical filters to visualize the red fluorescence of PpIX and thereby detect tumorous tissue. Conventional PpIX-guided surgery, however, is limited for lower-grade gliomas or low concentrations of PpIX in tumor infiltrated brain, where endogenous tissue fluorescence and parts of the blue excitation light outweigh the PpIX fluorescence $[3,15,16]$. Particularly, the vast majority of pure LGG lack fluorescence during resection [3,16-19], and visible fluorescence in these tumors was mostly observed in anaplastic foci $[17,20,21]$. Phase fluorescence lifetime imaging in frequency-domain (FD-FLIM) has shown promise for delineating low PpIX concentrations from competing fluorophores due to differences in their respective decay dynamics $[15,22-25]$. As excited-state populations of PpIX have a slower depopulation rate (native lifetime $\tau \approx 16.4 \mathrm{~ns}$ [26]), PpIX accumulation in tissue entails increased fluorescence lifetimes with respect to the approximately $1 \mathrm{~ns}$ to $2 \mathrm{~ns}$ found in physiological brain parenchyma $[15,24,25,27]$. When PpIX fluorescence and tissue autofluorescence are on the same order of magnitude, the measured lifetime depends on the relative PpIX and autofluorescence signal contributions [15]. The average FD-FLIM phase lifetime $\bar{\tau}_{\phi}$ for $i$ fluorophores contributing to a multiexponential decay is given by

$$
\bar{\tau}_{\phi}=\frac{\sum_{i} \alpha_{i} \tau_{i}^{2} /\left(1+\left(2 \pi f_{\text {mod }}\right)^{2} \tau_{i}^{2}\right)}{\sum_{i} \alpha_{i} \tau_{i} /\left(1+\left(2 \pi f_{\text {mod }}\right)^{2} \tau_{i}^{2}\right)},
$$

with $f_{\text {mod }}$ being the modulation frequency, $\alpha_{i}$ the intensity contribution and $\tau_{i}$ the fluorescence lifetime of each fluorophore $i$ [28]. In contrast, the average fluorescence lifetime measured in time-domain is given by

$$
\bar{\tau}=\frac{\sum_{i} \alpha_{i} \tau_{i}^{2}}{\sum_{i} \alpha_{i} \tau_{i}} .
$$

As a result, fluorophores with shorter lifetimes are weighted more strongly for frequency-domain measurements of the phase when compared to the average lifetime in time-domain FLIM [28]. For delineating weak concentrations of PpIX in tissue, it is therefore of particular interest to reduce autofluorescence contributions and maximize the PpIX signal within the detection band of the emission filter.

In our previous work, we employed a detection band from $590 \mathrm{~nm}$ to $740 \mathrm{~nm}$ for capturing the tissues' fluorescence response [15,22-25]. We hypothesize that adjusting the spectral detection band to optimize the PpIX to autofluorescence ratio further increases the overall measured lifetime, thus enhancing the contrast to the autofluorescence background of physiological brain parenchyma. To substantiate our hypothesis, both FD-FLIM and spectroscopic fluorescence data were acquired ex vivo on a set of 130 brain tumor specimens. We then evaluated both FD-FLIM and combined time- and spectrally resolved fluorescence data to verify the adjustments made to the detection band.

\section{Materials and Methods}

\subsection{FD-FLIM and Spectroscopy Imaging Setup}

FD-FLIM and spectroscopic data were acquired with a multimodal surgical microscope at a working distance of $200 \mathrm{~mm}$ (OPMI Visu 200, Carl Zeiss Meditec AG, Jena, Germany). The interested reader is referred to our previous work for a detailed description of the system $[15,23]$. In brief, fluorescence was excited by a $405 \mathrm{~nm}$ laser diode (phoxX-405, Omicron Laserage, Rodgau, Germany), which was modulated at a frequency of $10 \mathrm{MHz}$ and raster-scanned across the tissue. Fluorescence lifetime maps were acquired across a field of view of $6.5 \times 6.5 \mathrm{~mm}^{2}$ by employing a homodyne detection scheme. FD-FLIM acquisitions 
were performed in 16 seconds with a lateral resolution of $25 \mu \mathrm{m}(256 \times 256$ pixels $)$ at a laser power of $6 \mathrm{~mW}$. Our system allowed for steering the laser to specific regions of interest on the specimens to acquire complementary spectroscopic information. The imaging protocol can be found elsewhere [15]. Fluorescence emission was filtered by either a $590 \mathrm{~nm}$ to $740 \mathrm{~nm}$ (665/150 BrightLine HC Semrock, Rochester, NY, USA) bandpass filter or a $615 \mathrm{~nm}$ long-pass filter (ET615LP, Chroma, Bellow Falls, Vermont). We installed a multi-position slider with resonant piezoelectric motor (ELL9K, Thorlabs Inc., Newton, NJ, USA) for fast switching between the emission filters.

\subsection{Patient Cohort and Specimen Handling}

Brain tumor specimens were routinely and safely resected as part of an ongoing ex vivo study, kept in artificial cerebrospinal fluid (Landesapotheke Salzburg, 19C11S02) and imaged within one hour after resection. The histopathological workup was performed by experienced neuropathologists following the WHO classification of CNS tumors from 2016 [29]. In total, 14 LGG, 56 HGG, 39 MNG and 21 MET specimens were imaged with both filters and further grouped in subgroups, which were (1) samples without 5-ALA administration, (2) samples with 5-ALA administration but lack of visible fluorescence during surgery and (3) samples showing visible fluorescence during surgery. The group of MET specimens originated from different carcinomas, including bronchial, mammary, thyroid, urachal, renal cell and squamous cell carcinoma. The number of specimens with and without oral administration of 5-ALA considered in this study is given in Table 1. Specimens were imaged with both optical filters consecutively. For specimens with 5-ALA administration, we combined FD-FLIM with spectroscopic measurements. As the spectrometer channel was implemented at a later stage of the project, the number of specimens with spectroscopic measurements was 82 with a total of 269 data points (about 3 spectroscopic measurements per specimen). Approval by the ethics committee of the Medical University of Vienna (EK419/2008-Amendment 04/2018) was obtained, and patients were informed and gave written consent.

Table 1. Fluorescence lifetime statistics for metastases, low-grade gliomas, high-grade gliomas and meningiomas, measured with a $590 \mathrm{~nm}-740 \mathrm{~nm}$ bandpass and a $615 \mathrm{~nm}$ long-pass filter. The ex vivo imaged human brain tumor specimens were further grouped into samples without 5-ALA administration (No. 5-ALA) and samples with 5-ALA administration without or with visible intraoperative fluorescence (Fluo-, Fluo+, respectively).

\begin{tabular}{lcccccccccc}
\hline & \multicolumn{3}{c}{ Metastases } & \multicolumn{3}{c}{ Low-Grade Gliomas } & \multicolumn{2}{c}{ High-Grade Gliomas } & \multicolumn{2}{c}{ Meningiomas } \\
\hline Subgroup & No 5-ALA & Fluo- & Fluo+ & No 5-ALA & Fluo- & Fluo- & Fluo+ & No 5-ALA & Fluo- & Fluo+ \\
\hline filter & & & & & 590 mm-740 & mm/>615 nm & & & \\
N samples & $6 / 6$ & $5 / 5$ & $10 / 10$ & $4 / 4$ & $10 / 10$ & $19 / 19$ & $37 / 37$ & $20 / 20$ & $3 / 3$ & $16 / 16$ \\
median $\tau / \mathrm{ns}$ & $2.5 / 2.5$ & $4.3 / 4.9$ & $8.4 / 9.8$ & $2.3 / 2.4$ & $4.7 / 5.4$ & $2.9 / 3.3$ & $13.4 / 14.1$ & $2.4 / 2.2$ & $4.2 / 3.8$ & $13.2 / 13.8$ \\
Q1 $\tau / \mathrm{ns}$ & $2.3 / 2.1$ & $4.2 / 4.8$ & $7.7 / 8.8$ & $1.9 / 1.9$ & $4.0 / 4.6$ & $2.5 / 2.7$ & $12.2 / 13.0$ & $2.2 / 2.0$ & $3.7 / 3.4$ & $11.0 / 12.1$ \\
Q2 $\tau / \mathrm{ns}$ & $3.0 / 3.3$ & $4.4 / 5.0$ & $9.7 / 11.3$ & $3.0 / 3.3$ & $5.5 / 6.2$ & $3.6 / 4.5$ & $14.1 / 14.6$ & $3.0 / 2.7$ & $4.5 / 4.7$ & $14.2 / 14.6$ \\
$p$-value & 0.47 & 0.03 & 0.11 & 0.44 & 0.17 & 0.18 & 0.04 & 0.9 & 0.67 & 0.15 \\
\hline
\end{tabular}

\section{Results}

\subsection{Optimizing the FD-FLIM Detection Band for Enhanced Delineation of PpIX from Tissue Autofluorescence}

When detecting weak PpIX concentrations in fluorescence-guided surgery, the autofluorescence background of the brain can be stronger than the actual PpIX fluorescence. This is illustrated with a representative LGG shown in Figure 1. Upon excitation with $405 \mathrm{~nm}$, the fluorescence of the specimen captured with a scientific camera $(>430 \mathrm{~nm})$ was white to faint blue (aI). Tissue autofluorescence dominated the overall signal, and the red PpIX fluorescence could not be observed visually during surgical resection and imaging. Dark red spots were seen on areas covered with blood. Spectroscopic measurements (aII) were acquired on three selected ROIs, indicated through red squares. Small peaks at $635 \mathrm{~nm}$ 
and slightly below $700 \mathrm{~nm}$ were observed above the autofluorescence background, corresponding to weak accumulations of PpIX. A representative haematoxylin and eosin (H\&E) stain of the sample (aIII) showed CNS tissue with increased cellularity and infiltrating pleomorphic glial tumor cells. The emission band from $590 \mathrm{~nm}$ to $740 \mathrm{~nm}$, which we also employed in previous work, is illustrated in (bI). Signal contributions below the fitted line contributed to the autofluorescence background and were colored in blue. PpIX signal contributions were colored in red. The demodulated fluorescence intensity $\left(\mathrm{mV}_{R M S}\right)$ and lifetime (ns) measured with this filter are shown in (bII) and (bIII). While the mean fluorescence intensity across the sample was $8.8 \mathrm{mV}_{R M S}$, fluorescence lifetimes averaged over ROI 1 to 3 were $2.0 \mathrm{~ns}, 4.9$ ns and $3.7 \mathrm{~ns}$, respectively. In the measured PpIX emission spectra, a considerable amount of autofluorescence was observed at wavelengths between $590 \mathrm{~nm}$ and the actual PpIX emission peak. To determine the optimized cut-on wavelength employed in this work, we derived the fluorescence emission spectrum of PpIX (data taken from PhotochemCAD [30]) and chose the wavelength with an available off-the-shelf filter closest to the point where the derivation began to rise. This resulted in setting the cut-on at $615 \mathrm{~nm}$ as shown in Figure 1 (cI). Cutting away autofluorescence below $615 \mathrm{~nm}$ decreased the average fluorescence intensity to $7.1 \mathrm{mV}_{R M S}$ (cII). This in turn increased the relative contribution of PpIX to the overall signal. As a result, the average fluorescence lifetime of ROI 1 to 3 was increased to $2.2 \mathrm{~ns}, 5.6 \mathrm{~ns}$ and $4.3 \mathrm{~ns}$, respectively (cIII). On average, the signal intensity for detection $>615 \mathrm{~nm}$ was decreased by about $17 \%$ for all specimens when compared to the band from $590 \mathrm{~nm}$ to $740 \mathrm{~nm}$.

$\mathbf{a}$
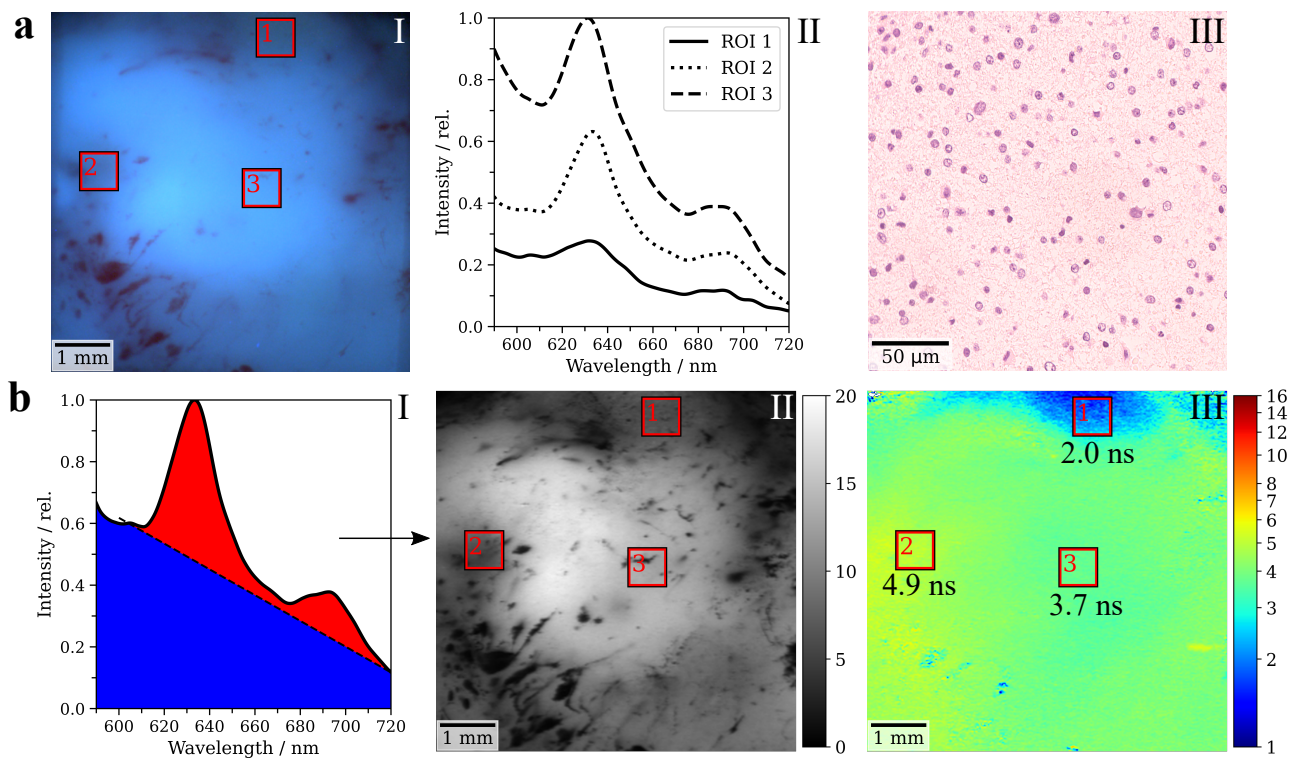

C
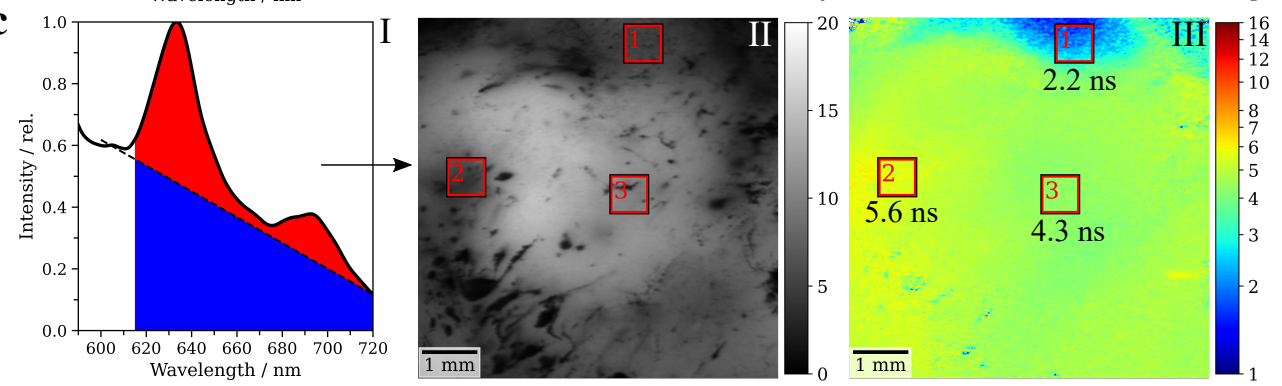

Figure 1. (a) Fluorescence $>430 \mathrm{~nm}$ of the specimen captured with a scientific camera (I), spectra measured on specific regions of interest on the specimen (II), and representative histopathological H\&E stained scan (III). Data were acquired with an emission filter from 590 to $740 \mathrm{~nm}(\mathbf{b}(\mathrm{I}))$ and $>615 \mathrm{~nm}(\mathrm{c}(\mathrm{I}))$. The respective demodulated root mean square fluorescence intensity $\left(\mathrm{mV}_{R M S}\right)$ and lifetime (ns) maps are shown in (b(II),c(II)) and (b(III),c(III)). 


\subsection{Minimizing Autofluorescence Crosstalk Improves the Detection of Protoporphyrin IX with Frequency-Domain FLIM}

Based on these observations, we conducted a study to investigate the effect of the adjusted filter on a larger number of specimens from various tumor entities. We acquired FD-FLIM measurements on 130 brain tumor specimens ex vivo and combined them with additional spectroscopic measurements. Spectra were used to determine the relative PpIX signal contribution $\left(\mathrm{RSC}_{P p I X}\right)$ [15], which is why specimens without 5-ALA administration were excluded from the analysis in Figure 2a.

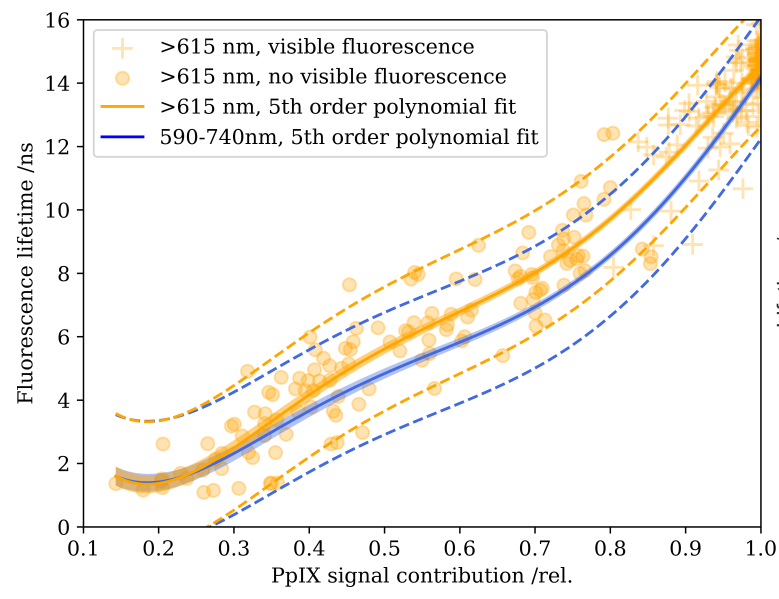

c

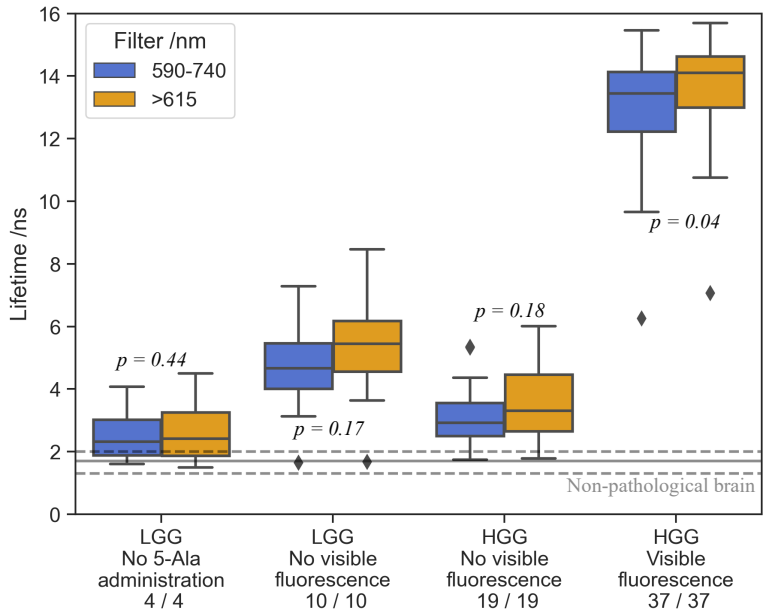

b
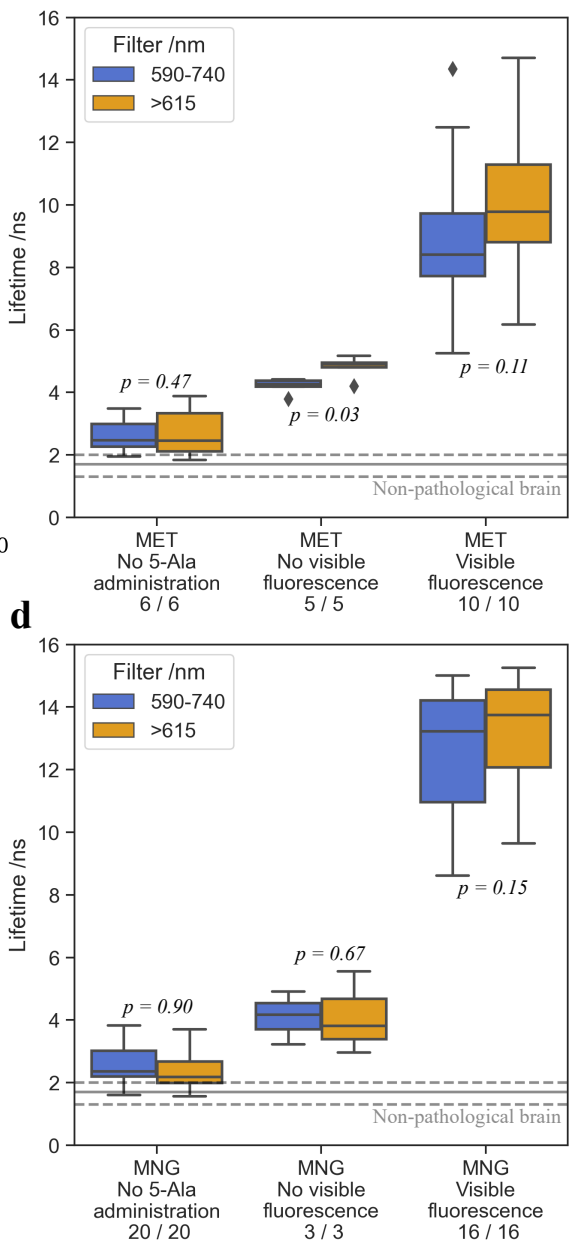

Figure 2. Comparison of FD-FLIM PpIX detection for the spectral bands $590 \mathrm{~nm}$ to $740 \mathrm{~nm}$ and $>615 \mathrm{~nm}$. (a) Correlating the fluorescence lifetime with the relative PpIX signal contribution illustrates an increase in the measured lifetime for detection $>615 \mathrm{~nm}$ starting around 0.25 relative PpIX signal contribution. 95\% prediction intervals (dashed lines) and 95\% confidence intervals (color shade around the 5th order polynomial fit) are given both for detection from 590-740 nm and $>615 \mathrm{~nm}$. Fluorescence lifetimes were then compared for the two bands for metastases (b), low-and high-grade gliomas (c) and meningiomas (d). Samples were grouped according to the perceived fluorescence during surgery (visible, not visible), and samples without 5-ALA administration prior to surgery were treated as a separate group. Data from non-pathological specimens from our previous work [15] are indicated with gray lines (solid: median, dashed: first and third quartiles) for direct comparison to the lifetimes measured in tumorous tissue. The number of specimens per filter and subgroup is indicated at the lower end of (b-d) (e.g., 6/6).

Plotting the measured lifetime as a function of the $\mathrm{RSC}_{P p I X}$ illustrated how blocking autofluorescence below $615 \mathrm{~nm}$ impacted the measured lifetime (Figure 2a). Data acquired with the $615 \mathrm{~nm}$ long-pass filter were drawn in yellow. Data points were labelled according 
to their fluorescence status during surgery, with a circle indicating lack of visibility and a plus visible fluorescence. Below 0.25 RSC $C_{P p I X}$, autofluorescence dominated the overall signal with close to no alterations of the measured fluorescence lifetime between both filters. Above $0.25 \mathrm{RSC}_{P p I X}$, the measured lifetime began to increase due to the increased PpIX content in the signal. Comparing the two filters, we observed a stronger increase in the measured lifetime for the cut-on at $615 \mathrm{~nm}$, gradually increasing from $0.25 \mathrm{RSC}_{P p I X}$ to about 0.8 RSC $_{P p I X}$. With PpIX fluorescence and tissue autofluorescence being on the same order of magnitude, this filter impacted the fluorophore ratio in favor of PpIX. Above $0.8 \mathrm{RSC}_{P p I X}$, PpIX fluorescence gradually started to become visible during surgical resection, with PpIX now being the dominant signal contributor. As a result, tissue autofluorescence had less influence on the overall signal and the differences of the measured lifetimes between both filters began to decrease. Samples with strong intraoperative fluorescence were condensed at a RSC $P$ PIX of 1.0. We then grouped all specimens in MET (Figure 2b), LGG, HGG (Figure 2c) and MNG (Figure 2d). For each of the groups, we further distinguished between specimens where no 5-ALA was administered prior to surgery, specimens with 5-ALA administration but no visible fluorescence during surgery and samples with visible fluorescence during surgery. Median as well as 0.25 and 0.75 quartiles were visualized with box plots for all subgroups. As expected, the $615 \mathrm{~nm}$ long-pass filter led to an increase of the measured lifetime for most subgroups with 5-ALA administration. Median lifetimes in LGG specimens were $5.4 \mathrm{~ns}$ and $4.7 \mathrm{~ns}$ for the $615 \mathrm{~nm}$ long-pass and the $590 \mathrm{~nm}$ to $740 \mathrm{~nm}$ bandpass filters, respectively. However, no significant differences were found between the two emission filters $(p=0.17)$. Similarly, median lifetimes were increased for HGG without ( $3.3 \mathrm{~ns}, 2.9 \mathrm{~ns}, p=0.18)$ and with $(14.1 \mathrm{~ns}, 13.4 \mathrm{~ns}, p=0.04)$ visible fluorescence. This was also observed in MET without visible fluorescence ( $4.9 \mathrm{~ns}, 4.3 \mathrm{~ns}, p=0.03)$ and visible fluorescence ( $9.8 \mathrm{~ns}, 8.4 \mathrm{~ns}, p=0.11)$. Only in the MNG group did specimens lacking visible fluorescence have lower lifetimes for the $615 \mathrm{~nm}$ longpass filter ( $3.8 \mathrm{~ns}, 4.2 \mathrm{~ns}, p=0.67$ ), which will be discussed later on. Lifetimes in specimens from patients without 5-ALA administration were barely affected by the filter change in MET $(2.5 \mathrm{~ns}, 2.5 \mathrm{~ns}, p=0.47)$ and LGG (2.4ns, $2.3 \mathrm{~ns}, p=0.44)$, and even decreased in MNG (2.2 ns, $2.4 \mathrm{~ns}, p=0.90)$. Please refer to Table 1 for complete data of all subgroups. Lifetimes measured in non-pathological brain from our previous work [15] are indicated with gray lines (solid: median, dashed: first and third quartiles). Specimens lacking visible fluorescence during surgery could be distinguished from this autofluorescence baseline with both filters. Yet, the optimized filter increased the measured lifetimes in most subgroups, which benefits the detection of weak PpIX accumulations.

\section{Discussion}

In this work, we improved FD-FLIM PpIX visualization for fluorescence-guided surgery by minimizing autofluorescence crosstalk within the detection band. Optical filters have to be tailored to their respective application to ensure optimal performance in fluorescence imaging. This is of importance, especially when several fluorophores are excited and emitting within the detection band. In metabolic imaging of the intrinsic fluorophores nicotinamide adenine dinucleotide (phosphate) $(\mathrm{NAD}(\mathrm{P}) \mathrm{H})$ and flavin adenine dinucleotide (FAD), substantial work has been performed to optimize the filter specifications and minimize crosstalk [31,32]. FLIM of PpIX has emerged only recently, and previous work has employed a broad detection band $(590-740 \mathrm{~nm})$ to capture the full PpIX emission spectrum and to increase the overall collected signal [15,22-25]. This did not fully consider the influence of tissue autofluorescence, which can be of the same order of magnitude as PpIX fluorescence for lower-grade tumors and weakly tumor-infiltrated brains. In FD-FLIM, the measured lifetime is a weighted average of all emitting fluorophores. When exciting fluorescence in the blue range of the spectrum, tissue autofluorescence of non-pathological brain above $590 \mathrm{~nm}$ decays in about $1-2 \mathrm{~ns}[15,24,25,27]$, with individual contributions of flavins, lipofuscin, vitamin A, endogenous porphyrins and further fluorophores [33]. Autofluorescence lifetimes in tumorous tissue were found to be increased beyond $2 \mathrm{~ns}$ [34]. 
PpIX yields higher lifetimes with up to $16.4 \mathrm{~ns}$ in organic solvent [26] and about $6.3 \mathrm{~ns}$ [26] to $7.4 \mathrm{~ns}$ [35] in mitochondria. Photoprotoporphyrin and the photoproducts II+III decay within $5.4 \mathrm{~ns}$ in mitochondria [36] and about 2-4 ns in the cytoplasm [37], respectively.

Weak concentrations of PpIX in tissue can be detected by increased lifetimes with respect to the lifetimes of the autofluorescence background. A higher relative contribution of PpIX to the overall excited state population thereby increases the average lifetime of all fluorophores contributing to the multi-exponential decay [15]. Combining FD-FLIM with optical spectroscopy revealed a stronger increase of the measured lifetime starting at a RSC $_{P p I X}$ of about 0.25 when detecting fluorescence $>615 \mathrm{~nm}$. Blocking autofluorescence between $590 \mathrm{~nm}$ and $615 \mathrm{~nm}$ increased the contribution of PpIX to the overall signal, which favored distinguishing PpIX from the autofluorescence background. Similarly, descriptive statistics coherently showed increased lifetimes for all subgroups with 5-ALA administration, besides the MNG group lacking visual fluorescence during resection. For this group, we observed increased autofluorescence signal contributions in our spectral measurements. A potential explanation could be the accumulation of flavins with relatively long lifetimes, such as flavin mononucleotide ( $4.8 \mathrm{~ns}$ when bound to proteins). Removing these contributions with the $615 \mathrm{~nm}$ longpass filter would explain the contrary effect on the overall lifetime for MNG with low PpIX concentrations. Further studies investigating on the autofluorescence properties, especially of MNG, are warranted. Differences between the two spectral filters were not found to be significant for most of the groups, which can be contributed to a limited number of samples per group and a relatively high variance within the groups compared to the lifetime differences between the groups. Although 130 specimens were included in the study, the number of tumor entities and subgroups (visible-, not visible fluorescence, and no 5-ALA administration) further reduced the group size. Additionally, specimens from core tumor areas, infiltration zones, and necrotic and reactive areas were combined within the subgroups, which contributed to the variance within groups. Yet, the trend of increased fluorescence lifetimes for the optimized filter was very coherent for all subgroups, with the exemption of MNG group discussed above. Note also that the reduced overall signal intensity in the optimized filter (17\% less compared to the previous filter) may contribute to an increase of phase noise and thereby a reduction of the signal-to-noise ratio for tissue exhibiting very weak fluorescence, e.g., parts of the specimens covered with blood. The overall photon budget, however, is mainly determined by the detection efficiency of the system and could be tailored to an acceptable level of phase noise. The influence of the integrated signal on noise in FD-FLIM phase measurements is described in more detail in the supplementary materials of our previous work [15]. We furthermore emphasize that of the 37 specimens throughout all tumor entities lacking visible fluorescence during surgery, all specimens could be distinguished from the $1 \mathrm{~ns}$ to $2 \mathrm{~ns}$ expected for non-pathological brain by increased fluorescence lifetimes.

Note that imaging of specimens to compare filters had to be performed consecutively, which might have led to bleaching of fluorescence during the first measurement and affected the second acquisition. We analyzed bleaching by consecutively imaging a specimen with the same filter and found that bleaching-induced lifetime reduction between two scans was smaller than $0.1 \mathrm{~ns}$. Additionally, we varied the order of which filter was used to image first between specimens and could not find any influence on the imaging order.

The overall coherence of what was observed in all groups and the multimodal FDFLIM and spectroscopy evaluation provided evidence to favor the $615 \mathrm{~nm}$ long-pass filter. Potential further optimization of the PpIX to autofluorescence ratio would be to restrict the detection band to the main PpIX emission peak. We performed a first test with a $620 \mathrm{~nm}$ to $640 \mathrm{~nm}$ bandpass filter, which slightly increased the measured lifetime with respect to to the $615 \mathrm{~nm}$ long-pass filter at the cost of a reduced signal to noise ratio and increased phase noise in our measurements. Additionally, a two-peaked emission of PpIX was observed in LGG, with one physicochemical state peaking at a lower wavelength of $620 \mathrm{~nm}$ [38]. Hence, a cut-on wavelength at $615 \mathrm{~nm}$ was the preferred choice. While we employed an off-the-shelf long-pass filter, a bandpass filter with a cut-off at around 
$715 \mathrm{~nm}$, which is right after the second PpIX emission peak, seems to be the optimal choice for FD-FLIM imaging of PpIX. Spectrally resolved FLIM would be highly beneficial to investigate this in more detail [39]. A valuable additional frequency-domain metric in future studies would be the modulation lifetime $\tau_{m}$ [28]. Thus, a measure of the ground-state heterogeneity could be given by $\left(\tau_{m} / \tau_{\phi}\right)-1$, essentially reflecting the lifetime variance of the multi-exponential fluorophore decays within the detected spectrum. For higher-grade tumors, where PpIX fluorescence outweighs tissue autofluorescence, this measure would be expected to approach 0 as $\tau_{\phi}=\tau_{m}$ for mono-exponential decays [28]. For lower-grade tumors, where PpIX is on the order of magnitude of tissue autofluorescence, the ground state heterogeneity could be an interesting additional metric for distinguishing 5-ALA labeled tumors from non-pathological brain. Additionally, a lifetime based ratio of PpIX to background fluorescence could be given, e.g., by transforming phase and modulation lifetime to the vector space of phasor plots [24,40]. Furthermore, time-domain FLIM would allow for better contrasting PpIX from the autofluorescence background by fitting a multiexponential model. Fluorescence anisotropy measurements of rotational correlation time potentially would provide an additional source of contrast for resolving ground state heterogeneity in 5-ALA labeled tumors and non-pathological brain. Yet, as outlined in this and our previous works [15,22-25], the average phase lifetime in FD-FLIM alone does constitute a sensitive and promising approach for PpIX fluorescence guidance.

In conclusion, we showed that FD-FLIM for PpIX-guided brain tumor visualization can be further improved by minimizing autofluorescence crosstalk in the detection band. Fluorescence should be detected with a cut-on wavelength of $615 \mathrm{~nm}$ in future systems and studies for obtaining optimal contrast between weak PpIX concentrations in lower-grade tumors or weakly tumor infiltrated brain with respect to the autofluorescence background of non-pathological brain.

Author Contributions: G.W., B.K. and L.I.W. resected tumor specimens. J.G., T.R.-P. and A.W. performed the histopathological workup. B.K., L.I.W. and A.L. organized biopsy handling and preparation. M.W. and C.H. provided technical consultation for modifications on the surgical microscope. M.T.E. and D.R. set up the FD-FLIM system under supervision of A.U. and M.A. D.R. implemented the spectroscopy channel, performed the measurements, analyzed the data and wrote the manuscript. M.T.E., W.D., G.W. and R.A.L. initiated the project. All authors have read and agreed to the published version of the manuscript.

Funding: This project has received funding from the Austrian Christian Doppler Research Association as well as from the innovation board of the Carl Zeiss Meditec AG. T.R. is recipient of a DOC Fellowship of the Austrian Academy of Sciences at the Division of Neuropathology and Neurochemistry (25262). The financial support by the Austrian Federal Ministry for Digital and Economic Affairs and the National Foundation for Research, Technology and Development is gratefully acknowledged. This project has furthermore received funding from the European Union Horizon 2020 research and innovation program under the Marie Sklodowska-Curie grant agreement (MSCA grant 721766) and from the OeNB grant 16725.

Institutional Review Board Statement: The study was conducted according to the guidelines of the Declaration of Helsinki and approved by the Ethics Committee of the Medical University of Vienna (EK419/2008-Amendment 04/2018).

Informed Consent Statement: Informed consent was obtained from all subjects involved in the study.

Data Availability Statement: The datasets generated and analyzed during this study are available from the corresponding author on reasonable request.

Acknowledgments: We thank Tanja Peilnsteiner for consistent support with biopsy preparation.

Conflicts of Interest: M.W. and C.H. are employees of the Carl Zeiss Meditec AG, Oberkochen, Germany. G.W. received restricted travel grants from NX Development Corp. All other authors declare to have no conflicts of interest. 


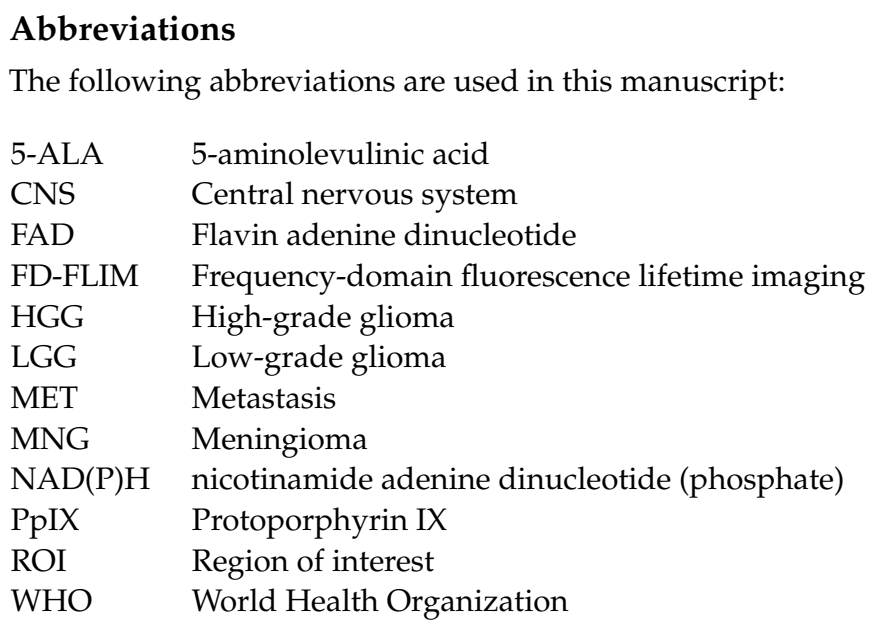

\section{References}

1. Ostrom, Q.T.; Patil, N.; Cioffi, G.; Waite, K.; Kruchko, C.; Barnholtz-Sloan, J.S. CBTRUS statistical report: Primary brain and other central nervous system tumors diagnosed in the United States in 2013-2017. Neuro-Oncology 2020, 22, iv1-iv96. [CrossRef]

2. Stupp, R.; Mason, W.P.; Van Den Bent, M.J.; Weller, M.; Fisher, B.; Taphoorn, M.J.; Belanger, K.; Brandes, A.A.; Marosi, C.; Bogdahn, U.; et al. Radiotherapy plus concomitant and adjuvant temozolomide for glioblastoma. N. Engl. J. Med. 2005, 352, 987-996. [CrossRef] [PubMed]

3. Widhalm, G.; Olson, J.; Weller, J.; Bravo, J.; Han, S.J.; Phillips, J.; Hervey-Jumper, S.L.; Chang, S.M.; Roberts, D.W.; Berger, M.S. The value of visible 5-ALA fluorescence and quantitative protoporphyrin IX analysis for improved surgery of suspected low-grade gliomas. J. Neurosurg. 2019, 133, 79-88. [CrossRef] [PubMed]

4. Xia, L.; Fang, C.; Chen, G.; Sun, C. Relationship between the extent of resection and the survival of patients with low-grade gliomas: A systematic review and meta-analysis. BMC Cancer 2018, 18, 48. [CrossRef] [PubMed]

5. Hervey-Jumper, S.L.; Berger, M.S. Role of surgical resection in low-and high-grade gliomas. Curr. Treat. Opt. Neurol. 2014, 16, 284. [CrossRef]

6. Sanai, N.; Polley, M.Y.; McDermott, M.W.; Parsa, A.T.; Berger, M.S. An extent of resection threshold for newly diagnosed glioblastomas. J. Neurosurg. 2011, 115, 3-8. [CrossRef] [PubMed]

7. Aizer, A.A.; Bi, W.L.; Kandola, M.S.; Lee, E.Q.; Nayak, L.; Rinne, M.L.; Norden, A.D.; Beroukhim, R.; Reardon, D.A.; Wen, P.Y.; et al. Extent of resection and overall survival for patients with atypical and malignant meningioma. Cancer 2015, 121, 4376-4381. [CrossRef]

8. Hess, K.R. Extent of resection as a prognostic variable in the treatment of gliomas. J. Neuro-Oncol. 1999, 42, 227-231. [CrossRef]

9. Schödel, P.; Schebesch, K.M.; Brawanski, A.; Proescholdt, M.A. Surgical resection of brain metastases-Impact on neurological outcome. Int. J. Mol. Sci. 2013, 14, 8708-8718. [CrossRef] [PubMed]

10. Stummer, W.; Pichlmeier, U.; Meinel, T.; Wiestler, O.D.; Zanella, F.; Reulen, H.J.; ALA-Glioma Study Group. Fluorescence-guided surgery with 5-aminolevulinic acid for resection of malignant glioma: A randomised controlled multicentre phase III trial. Lancet Oncol. 2006, 7, 392-401. [CrossRef]

11. Hadjipanayis, C.G.; Widhalm, G.; Stummer, W. What is the surgical benefit of utilizing 5-aminolevulinic acid for fluorescenceguided surgery of malignant gliomas? Neurosurgery 2015, 77, 663-673. [CrossRef] [PubMed]

12. Millesi, M.; Kiesel, B.; Mischkulnig, M.; Martínez-Moreno, M.; Wöhrer, A.; Wolfsberger, S.; Knosp, E.; Widhalm, G. Analysis of the surgical benefits of 5-ALA-induced fluorescence in intracranial meningiomas: Experience in 204 meningiomas. J. Neurosurg. 2016, 125, 1408-1419. [CrossRef] [PubMed]

13. Marhold, F.; Mercea, P.A.; Scheichel, F.; Berghoff, A.S.; Heicappell, P.; Kiesel, B.; Mischkulnig, M.; Borkovec, M.; Wolfsberger, S.; Woehrer, A.; et al. Detailed analysis of 5-aminolevulinic acid induced fluorescence in different brain metastases at two specialized neurosurgical centers: Experience in 157 cases. J. Neurosurg. 2019, 133, 1032-1043. [CrossRef] [PubMed]

14. Stepp, H.; Stummer, W. 5-ALA in the management of malignant glioma. Lasers Surg. Med. 2018, 50, 399-419. [CrossRef] [PubMed]

15. Reichert, D.; Erkkilae, M.T.; Gesperger, J.; Wadiura, L.I.; Lang, A.; Roetzer, T.; Woehrer, A.; Andreana, M.; Unterhuber, A.; Wilzbach, M.; et al. Fluorescence lifetime imaging and spectroscopic co-validation for protoporphyrin IX guided tumor visualization in neurosurgery. Front. Oncol. 2021, 11, 3613. [CrossRef] [PubMed]

16. Valdés, P.A.; Jacobs, V.; Harris, B.T.; Wilson, B.C.; Leblond, F.; Paulsen, K.D.; Roberts, D.W. Quantitative fluorescence using 5-aminolevulinic acid-induced protoporphyrin IX biomarker as a surgical adjunct in low-grade glioma surgery. J. Neurosurg. 2015, 123, 771-780. [CrossRef] [PubMed]

17. Ewelt, C.; Floeth, F.W.; Felsberg, J.; Steiger, H.J.; Sabel, M.; Langen, K.J.; Stoffels, G.; Stummer, W. Finding the anaplastic focus in diffuse gliomas: The value of Gd-DTPA enhanced MRI, FET-PET, and intraoperative, ALA-derived tissue fluorescence. Clin. Neurol. Neurosurg. 2011, 113, 541-547. [CrossRef] [PubMed] 
18. Sanai, N.; Snyder, L.A.; Honea, N.J.; Coons, S.W.; Eschbacher, J.M.; Smith, K.A.; Spetzler, R.F. Intraoperative confocal microscopy in the visualization of 5-aminolevulinic acid fluorescence in low-grade gliomas. J. Neurosurg. 2011, 115, 740-748. [CrossRef]

19. Jaber, M.; Ewelt, C.; Wölfer, J.; Brokinkel, B.; Thomas, C.; Hasselblatt, M.; Grauer, O.; Stummer, W. Is visible aminolevulinic acid-induced fluorescence an independent biomarker for prognosis in histologically confirmed (World Health Organization 2016) low-grade gliomas? Neurosurgery 2019, 84, 1214-1224. [CrossRef] [PubMed]

20. Widhalm, G.; Wolfsberger, S.; Minchev, G.; Woehrer, A.; Krssak, M.; Czech, T.; Prayer, D.; Asenbaum, S.; Hainfellner, J.A.; Knosp, E. 5-Aminolevulinic acid is a promising marker for detection of anaplastic foci in diffusely infiltrating gliomas with nonsignificant contrast enhancement. Cancer Interdiscip. Int. J. Am. Cancer Soc. 2010, 116, 1545-1552. [CrossRef] [PubMed]

21. Jaber, M.; Wölfer, J.; Ewelt, C.; Holling, M.; Hasselblatt, M.; Niederstadt, T.; Zoubi, T.; Weckesser, M.; Stummer, W. The value of 5-aminolevulinic acid in low-grade gliomas and high-grade gliomas lacking glioblastoma imaging features: An analysis based on fluorescence, magnetic resonance imaging, 18F-fluoroethyl tyrosine positron emission tomography, and tumor molecular factors. Neurosurgery 2016, 78, 401-411. [PubMed]

22. Erkkilä, M.T.; Bauer, B.; Hecker-Denschlag, N.; Madera Medina, M.J.; Leitgeb, R.A.; Unterhuber, A.; Gesperger, J.; Roetzer, T.; Hauger, C.; Drexler, W.; et al. Widefield fluorescence lifetime imaging of protoporphyrin IX for fluorescence-guided neurosurgery: An ex vivo feasibility study. J. Biophotonics 2019, 12, e201800378. [CrossRef] [PubMed]

23. Erkkilä, M.T.; Reichert, D.; Hecker-Denschlag, N.; Wilzbach, M.; Hauger, C.; Leitgeb, R.A.; Gesperger, J.; Kiesel, B.; Roetzer, T.; Widhalm, G.; et al. Surgical microscope with integrated fluorescence lifetime imaging for 5-aminolevulinic acid fluorescenceguided neurosurgery. J. Biomed. Opt. 2020, 25, 071202. [CrossRef]

24. Erkkilä, M.T.; Reichert, D.; Gesperger, J.; Kiesel, B.; Roetzer, T.; Mercea, P.A.; Drexler, W.; Unterhuber, A.; Leitgeb, R.A.; Woehrer, A.; et al. Macroscopic fluorescence-lifetime imaging of NADH and protoporphyrin IX improves the detection and grading of 5-aminolevulinic acid-stained brain tumors. Sci. Rep. 2020, 10, 20492. [CrossRef] [PubMed]

25. Reichert, D.; Erkkilä, M.T.; Holst, G.; Hecker-Denschlag, N.; Wilzbach, M.; Hauger, C.; Drexler, W.; Gesperger, J.; Kiesel, B.; Roetzer, T.; et al. Towards real-time wide-field fluorescence lifetime imaging of 5-ALA labeled brain tumors with multi-tap CMOS cameras. Biomed. Opt. Express 2020, 11, 1598-1616. [CrossRef] [PubMed]

26. Russell, J.A.; Diamond, K.R.; Collins, T.J.; Tiedje, H.F.; Hayward, J.E.; Farrell, T.J.; Patterson, M.S.; Fang, Q. Characterization of fluorescence lifetime of photofrin and delta-aminolevulinic acid induced protoporphyrin IX in living cells using single-and two-photon excitation. IEEE J. Sel. Top. Quantum Electron. 2008, 14, 158-166. [CrossRef]

27. Kantelhardt, S.R.; Diddens, H.; Leppert, J.; Rohde, V.; Hüttmann, G.; Giese, A. Multiphoton excitation fluorescence microscopy of 5-aminolevulinic acid induced fluorescence in experimental gliomas. Lasers Surg. Med. 2008, 40, 273-281. [CrossRef] [PubMed]

28. Lakowicz, J.R. Principles of Fluorescence Spectroscopy; Springer Science \& Business Media: Boston, MA, USA, 2013.

29. Louis, D.N.; Perry, A.; Reifenberger, G.; Von Deimling, A.; Figarella-Branger, D.; Cavenee, W.K.; Ohgaki, H.; Wiestler, O.D.; Kleihues, P.; Ellison, D.W. The 2016 World Health Organization classification of tumors of the central nervous system: A summary. Acta Neuropathol. 2016, 131, 803-820. [CrossRef] [PubMed]

30. PhotochemCAD 2: A Refined Program with Accompanying Spectral Databases for Photochemical Calculations. Photochem. Photobiol. 2005, 81, 212-213. [CrossRef]

31. Cao, R.; Wallrabe, H.K.; Periasamy, A. Multiphoton FLIM imaging of NAD (P) H and FAD with one excitation wavelength. J. Biomed. Opt. 2020, 25, 014510. [CrossRef] [PubMed]

32. Meyer, B.O.; Stella, M.P.J.; Holst, B.; Nielsen, B.S.; Holmstrøm, K.; Andersen, P.E.; Marti, D. Selecting optimal spectral bands for improved detection of autofluorescent biomarkers in multiphoton microscopy. J. Biomed. Opt. 2020, 25, 071206. [CrossRef] [PubMed]

33. Croce, A.C.; Bottiroli, G. Autofluorescence spectroscopy and imaging: A tool for biomedical research and diagnosis. Eur. J. Histochem. EJH 2014, 58, 2461. [CrossRef] [PubMed]

34. Kantelhardt, S.R. 20 Multiphoton microscopy and fluorescence lifetime imaging for resection guidance in malignant glioma surgery. In Multiphoton Microscopy and Fluorescence Lifetime Imaging; De Gruyter: Berlin, Germany, 2018; pp. 395-410.

35. Kress, M.; Meier, T.H.; Steiner, R.W.; Dolp, F.; Erdmann, R.; Ortmann, U.; Rueck, A.C. Time-resolved microspectrofluorometry and fluorescence lifetime imaging of photosensitizers using picosecond pulsed diode lasers in laser scanning microscopes. J. Biomed. Opt. 2003, 8, 26. [CrossRef] [PubMed]

36. Theodossiou, T.; MacRobert, A.J. Comparison of the Photodynamic Effect of Exogenous Photoprotoporphyrin and Protoporphyrin IX on PAM 212 Murine Keratinocytes. Photochem. Photobiol. 2002, 76, 530-537. [CrossRef]

37. Rück, A.; Dolp, F.; Hülshoff, C.; Hauser, C.; Scalfi-Happ, C. Fluorescence lifetime imaging in PDT. An overview. Med. Laser Appl. 2005, 20, 125-129. [CrossRef]

38. Montcel, B.; Mahieu-Williame, L.; Armoiry, X.; Meyronet, D.; Guyotat, J. Two-peaked 5-ALA-induced PpIX fluorescence emission spectrum distinguishes glioblastomas from low grade gliomas and infiltrative component of glioblastomas. Biomed. Opt. Express 2013, 4, 548-558. [CrossRef] [PubMed]

39. Williams, G.O.; Williams, E.; Finlayson, N.; Erdogan, A.T.; Wang, Q.; Fernandes, S.; Akram, A.R.; Dhaliwal, K.; Henderson, R.K.; Girkin, J.M.; et al. Full spectrum fluorescence lifetime imaging with $0.5 \mathrm{~nm}$ spectral and 50 ps temporal resolution. Nat. Commun. 2021, 12, 6616. [CrossRef]

40. Digman, M.A.; Caiolfa, V.R.; Zamai, M.; Gratton, E. The phasor approach to fluorescence lifetime imaging analysis. Biophys. J. 2008, 94, L14-L16. [CrossRef] 